\title{
Miniaturized Reaction Chamber for Optimized Laser-Assisted Carbon Nanotube Growth
}

\author{
Yoeri van de Burgt ${ }^{* 1,2}$, Wouter van Loon ${ }^{* 1}$, Rajesh Mandamparambil ${ }^{* 1,2}$, Yves Bellouard ${ }^{* 1}$ \\ ${ }^{* 1}$ Department of Mechanical Engineering, Eindhoven University Technology, Den Dolech 2, \\ Eindhoven, The Netherlands \\ E-mail: y.b.v.d.burgt@tue.nl \\ ${ }^{* 2}$ Holst Centre/TNO - Netherlands Organization for Applied Scientific Research, HTC31, \\ Eindhoven, The Netherlands.
}

\begin{abstract}
The localized growth of carbon nanotube structures has potential in many applications such as interconnects, field emitters and sensors. Using a laser to locally heat the substrate offers a highly versatile process compatible with a broad range of substrates and devices. However, for laserassisted CNT growth, detailed process information, such as temperature evolution and process monitoring over time are often unavailable. Here, we report on a miniaturized laser-CVD reactor, where a precise control of the gas flow and composition on the laser-growth site is provided. The particular design of this miniaturized reactor results in a high reproducibility as well as faster growth time. A multi-parameter finite element method (FEM) model is implemented to link substrate temperature at the laser spot with emitted radiation, taking into account the gas flow, the process time and other temperature-dependent physical parameters such as forced convection, thermal- conductivity and heat capacity. The resulting growth is assessed using Scanning Electron Microscopy. Combined with results from the thermal model, process information is used to calculate activation energy for the nanotube nucleation. Finally, several demonstrations of possible applications are given, exploiting the potentials of the miniaturized reaction chamber.
\end{abstract}

DOI: $10.2961 /$ jlmn.2014.02.0007

Keywords: Laser-assisted chemical vapor deposition, carbon nanotubes, miniaturized reactionchamber, thermal modeling, activation energy.

\section{Introduction}

Localized growth of carbon nanotube structures has potential in many applications such as field emitters [1], field effect transistors [2,3], filters [4], interconnects [5], and sensors [6]. A drawback of local growth using conventional CNT growth methods is the requirement for additional catalyst structuring steps and the high temperature applied on the whole substrate. Using a laser to locally heat the substrate offers a highly versatile process compatible with a broader range of substrates that can have various shapes or temperature-sensitive elements already attached to it. However, for laser-assisted CNT growth, detailed process information, such as temperature evolution and process monitoring over time are often unavailable, due to the nature of the localized process.

Recently, we have reported on a controlled local laserassisted CVD process for CNTs [7]. The process itself is monitored and controlled by different optical sensors, controlling temperature and monitoring the CNT growth. To further optimize the process, laser-CVD also requires a localized gas flow control, eliminating turbulence effects.

Here, we report on a miniaturized laser-CVD reactor (shown in Fig. 1), where a precise control of the gas flow on the laser-growth site is provided. The particular design of this miniaturized reactor results in a high reproducibility as well as a high production yield. A multi-parameter finite element model (FEM) is implemented to link substrate temperature at the laser spot with emitted radiation, taking into account gas flow, process time and other temperature- dependent physical parameters such as forced convection, thermal- conductivity and capacity.

To validate and calibrate the model, a series of testexperiments are performed. The thermal model then is applied to find a relation between reference radiation and temperature at the laser spot. The resulting CNT growth for certain process parameters is assessed using Raman spectroscopy and Scanning Electron Microscopy. To illustrate the versatility of the process, we demonstrate the growth of nanotubes in a narrow enclosure consisting of a U-shaped thick glass piece attached to the substrate as well as the ability to write lines by moving the whole chamber during synthesis.

\section{Design and Setup}

The necessity for a small reaction chamber originated from a limitation on the large, steady-state gas reaction chamber [7]. For accurate predictions on gas concentration around the laser hot-spot a laminar flow is required with the ability to control the gas flow. A miniaturized CVD reaction chamber was designed for this purpose, at the same time significantly reducing the experimental cycle time.

The laser-assisted chemical vapor deposition process for the growth of local carbon nanotube structures comprises of three main components. All three are essential to the growth of carbon nanotubes. Thermal energy, gas supply and catalyst are the building blocks for any CVD process. Here we use a CW short-IR laser $(808 \mathrm{~nm})$ to heat 


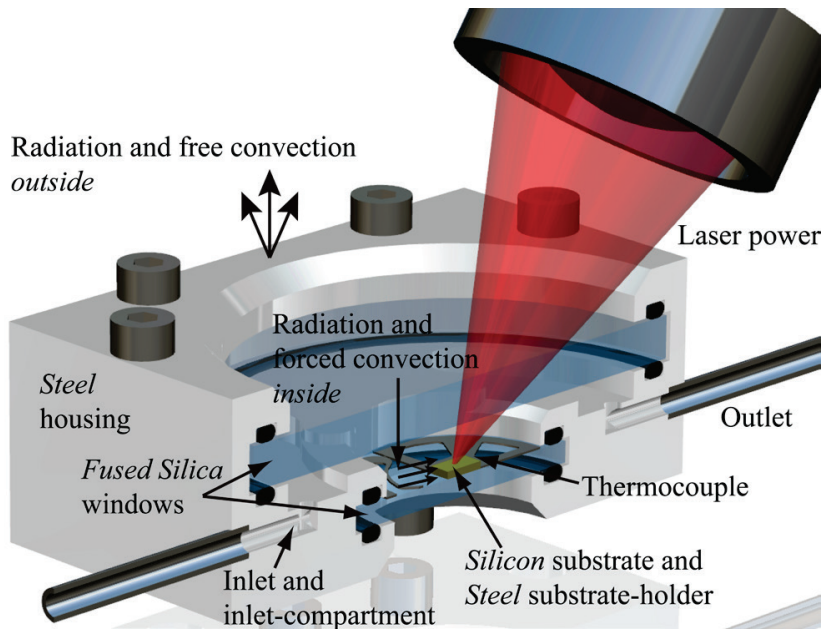

Fig. 1 CAD overview of half of the reaction chamber including the focused laser beam. Also indicated are the thermal properties and materials that are used in the FEM model as well as the position of the thermocouple used for model verification.

up the substrate. In our case the laser hits the substrate from the top surface. Although this method allows us to achieve higher spatial resolution for localized growth, it suffers from the fact that the substrate absorptivity dynamically evolves as CNTs start to grow. The enhanced absorbance during CNT growth results in overheating of the CNTs and amorphous carbon deposition [8]. To overcome this problem we have developed a closed-loop control synthesis method which uses the emitted radiation in the mid-IR spectrum from the laser hot-spot as the controller input [7]. This radiation is directly related to the temperature at the laser spot and enables us to have a better control over the process and prevent overheating. The growth itself is monitored by looking at the intensity variation of the reflected laser beam, providing a first approximation of the growth kinetics [9].

\subsection{Design}

The miniaturized reaction chamber design requirements comprised a laminar gas flow over the substrate and the ability to easily replace substrates and specimens while remaining optically accessible for the laser path and sensors. Further, by decreasing the size of the reactionchamber, the system can be placed onto an $x-y-z$ stage, enabling the creation of lines, patterns and arrays of CNT structures. The laser source is placed completely outside of the chamber. Taking advantage of its optical and thermal properties, fused silica was chosen as a covering top window. For similar reasons fused silica was also used as the bottom window. Especially, the low thermal conductivity and shock resistance of fused silica were proven to be essential for the growth process where the former enhances the thermal confinement.

To ensure a laminar flow, the reaction chamber was designed with an "inlet-compartment" to reduce any turbulence possibly present in the gas before entering the main reaction compartment of the chamber. The dimensions of the chamber ensure the Reynolds number does not exceed 35 for the maximum flow rates of the flow controller, significantly lower than 2000, where the transition to turbulent flow starts.
The chamber is sealed with flexible O-rings and clamped with bolts to avoid leaking. The substrate is held to its place by a substrate-holder fabricated by Electrical discharge machining (EDM) from stainless steel and comprises a small spring pushing the substrate on its place. The chamber is schematically shown in Fig 1.

\subsection{Experimental Setup}

The laser beam is focused through the window onto the substrate under an angle of $45^{\circ}$ and the reflection signal is captured by the $\mathrm{Si}$-detector under the same angle. The chamber is placed on an $x-y-z$ stage by an extended clamping plate to ensure the bottom window is visible by the radiation detector from beneath the chamber. The laser diode is connected to the computer by a data acquisition board enabling real-time control over the laser irradiance. Simulink is used to create a PID-controller that controls the radiation. The process gases, argon, hydrogen and ethylene can be individually controlled with a maximum flow rate of $500 \mathrm{sccm}$ (standard cubic centimeter per minute).

\section{Thermal modeling}

A finite element method (FEM) model is developed in COMSOL Multiphysics 4.3a. This thermal model is necessary in order to investigate temperature evolution in time and space. In this particular design, heat and flow are strongly coupled. Here our model evaluates forced and natural convection as a function of input parameters such as gas flow and laser irradiance.

The miniaturized reaction chamber designed here allows for the heat and flow problems to be coupled so that heat transfer through forced and natural convection is calculated by the model itself. This means that the input gas flow and the concentration will be directly related to the calculated temperature and vary for different input conditions.

All the materials and gases described in the model have temperature dependent properties. That is, heat capacity, thermal conductivity, and thermal diffusivity for the gases; heat capacity, thermal conductivity and thermal expansion for the solids. Gases are modeled using the ideal gas law, which is assumed to be valid within the pressure and temperature range considered here. The inlet and outlet boundary conditions are prescribed mass flow - flow rate and laminar outflow - pressure, respectively. Further, on all exterior and interior free boundaries surface radiation is applied and natural convection in air is applied on the exterior free boundaries. The laser is modeled as an ellipse with radii $350 \times 500 \mu \mathrm{m}$ and a Gaussian intensity profile and can be stationary, in the case of open-loop experiments, or time-dependent, in the case of closed-loop experiments. The laser is irradiance is applied as a boundary heat source and for the latter the recorded laser irradiance in time is used as the input instead of a constant laser irradiance.

\subsection{Experimental validation of the model}

To validate the model, two different flow conditions (flow of Argon of $50 \mathrm{sccm}$ and $500 \mathrm{sccm}$ ) have been selected and the corresponding temperature is calculated for a range of laser irradiances. Since measuring the temperature directly in the laser spot is not possible, a 
Table 1 Stationary temperature at edge substrate for different laser irradiances and flow conditions

\begin{tabular}{crcrr}
\hline $\begin{array}{c}\text { Irradiance } \\
\left(\mathrm{W} / \mathrm{mm}^{2}\right)\end{array}$ & $\begin{array}{r}\text { Ar flow } \\
(\mathrm{sccm})\end{array}$ & Exp. & Model & $\begin{array}{r}\text { Rel. error } \\
(\%)\end{array}$ \\
\hline 9.7 & $500 \mathrm{sccm}$ & $278{ }^{\circ} \mathrm{C}$ & $241{ }^{\circ} \mathrm{C}$ & -13.4 \\
\hline & $50 \mathrm{sccm}$ & $303{ }^{\circ} \mathrm{C}$ & $275{ }^{\circ} \mathrm{C}$ & -9.2 \\
17.9 & $500 \mathrm{sccm}$ & $429{ }^{\circ} \mathrm{C}$ & $406{ }^{\circ} \mathrm{C}$ & -5.4 \\
\hline & $50 \mathrm{sccm}$ & $472{ }^{\circ} \mathrm{C}$ & $454{ }^{\circ} \mathrm{C}$ & -3.8 \\
\hline 26 & $500 \mathrm{sccm}$ & $546{ }^{\circ} \mathrm{C}$ & $548{ }^{\circ} \mathrm{C}$ & 0.4 \\
\hline & $50 \mathrm{sccm}$ & $583{ }^{\circ} \mathrm{C}$ & $605{ }^{\circ} \mathrm{C}$ & 3.8 \\
\hline
\end{tabular}

thermocouple has been placed at the edge of the substrate. Stationary temperature is measured when the substrate is exposed to various preselected laser irradiance levels. The results are shown in Table 1. It appears that the model slightly over-estimates the temperature at high laser power and under-estimates the temperature at lower laser power. This effect seems to be larger for the lower flow rate of 50 sccm. The discrepancy can be explained by a number of factors. Most importantly, the calculation is done on a highly complex multi-parameter and multi-physics model and therefore the coupling between the forced gas flow and the thermal heat transfer in solids is very sensitive. It could be that the gas inlet flow differs somewhat in reality, changing the kinetics and ultimately the temperature. Furthermore, we assume an ideal gas which is reasonable under the current conditions but not perfect. Despite the complexity of the model and the multiple approximations made, the results show a rather good agreement between the measured temperature and the predicted one with a relative error between -13 and $4 \%$.

\section{Results}

Our miniaturized reaction chamber, in combination with the closed-loop control system results in fast, temperature-controlled growth of carbon nanotubes with a precise known gas composition. These benefits can be demonstrated by comparing the closed-loop process with the open-loop case.

\subsection{Experimental results}

To evaluate and quantify the temperature response of the system, the thermal model is used to compare the closed-loop control system operation with an open-loop one. The results are plotted in Fig 2. As the emitted radiation is used as the controlled variable this signal (black line) is plotted together with the reference set-point (black dashed line). The particular choice of the PIDcontroller values results in this case in an over-damped response, without any overshoot. The result is that the radiation set-point is reached after about 15 seconds. A similar response is found in the model, when looking at the temperature at the edge of the substrate (red line). The real values measured by thermocouple at certain times (red squares) are compared with the model and correspond well. The temperature in the center of the laser-spot (blue line) shows a small overshoot but settles around 12 seconds as well. The nature of the overshoot is not fully understood.

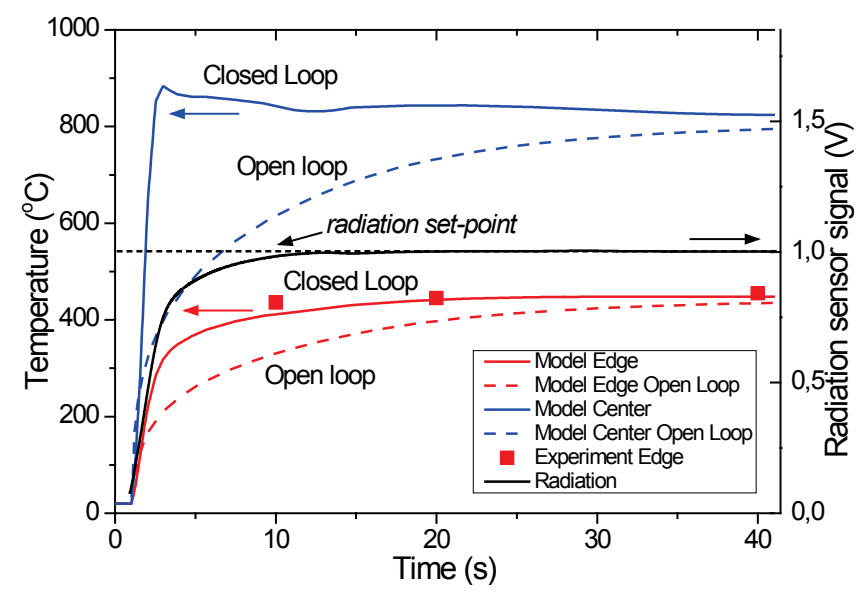

Fig. 2 Modeled temperature as a function of time in closed-loop setup compared with modeled temperature in open-loop. Also plotted is the radiation in volts as a function of time. This radiation is the controlled parameter.

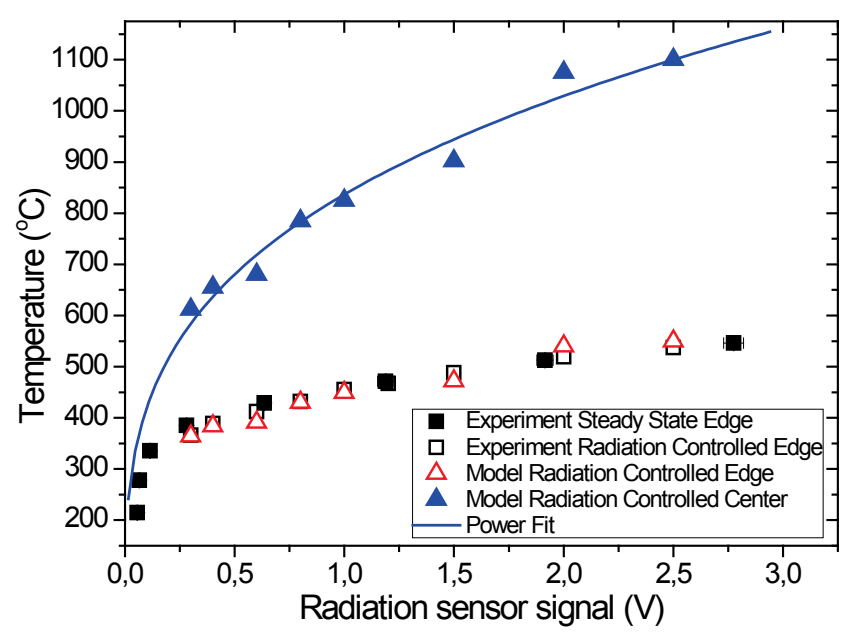

Fig. 3 Modeled temperature as a function of set-point radiation. The temperatures at the center of the spot (blue triangles) are fitted with a power law. The temperature at the edge of the substrate is give both by experimentally measuring for open-loop (black filled squares) and closed-loop (black open squares) as well as the modeled temperature (red open triangle).

We assume that the high sensitivity to the input power over time as well as the still simplified model and assumptions could account for the deviation from reality. The time-step used in the FEM solution might be too large and the real, non-linear response to an instant laser pulse is difficult to model. This assumption is to some extent confirmed by the temperature response at the edge of the substrate. This position is further from the spot and is thus less affected by near non-linear effects. The smooth temperature response, very similar to the radiation signal confirms this.

To find a direct relation between the radiation set-point and temperature, closed-loop experiments were carried out for a range of different radiation set-points. Similar responses as shown in Fig 2 were found and the steadystate temperatures of both the center at the laser spot as well as at the edge of the substrate are plotted against the set-point radiation. This is shown in Fig 3. The triangles (blue and red) represent the modeled temperature at the 

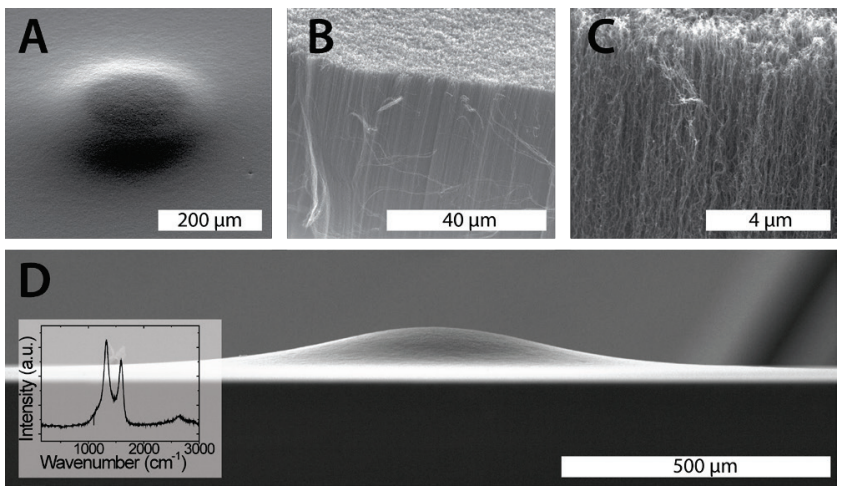

Fig. 4 SEM Micrographs of a localized hill of vertically aligned carbon nanotube forest created with the miniaturized reaction chamber. A. Overview. B. Zoom of the aligned nanotubes after scratching the surface. C. Enhanced zoom of the top of the nanotubes. D. Side view. Inset shows Raman intensity signal and indicates multi-walled nanotubes.

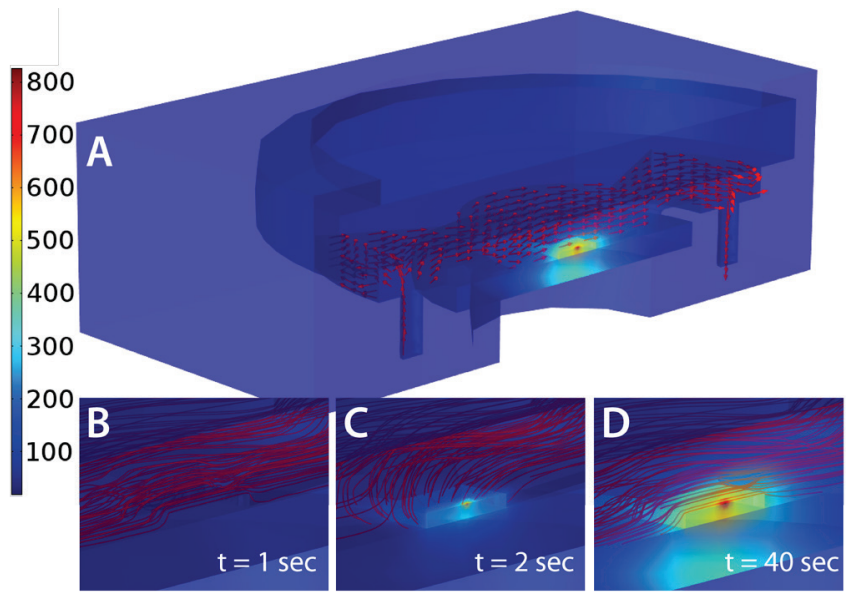

Fig. 5 Finite Element Modeling results depicted for different times. The red lines are stream lines while the colors depict temperature. A. Overview of half of the chamber. B. Zoom of the substrate with flow streamlines at $1 \mathrm{sec}$. C. Zoom of the substrate just after laser has been switched on. The flow streamlines show a sudden increase in pressure which affects the flow direction. D. Zoom of the substrate after $40 \mathrm{sec}$. The flow has been normalized again.

center of the laser spot and the edge of the substrate, respectively. To further validate the thermal model, the measured temperature at the edge of the substrate is plotted in this figure. The filled black squares are temperatures obtained with an open-loop experiment reaching a steadystate value and the corresponding emitted radiation. The open squares represent the temperature of the radiationcontrolled experiments, measured by thermocouple.

Using the miniaturized reaction chamber, with a radiation-controlled temperature of $850^{\circ} \mathrm{C}$ and flow rates of 200, 250 and $50 \mathrm{sccm}$, for argon, ethylene and hydrogen, respectively we were able to grow a localized hill of aligned carbon nanotubes, as depicted in Fig 4.

\subsection{Flow Simulation Results}

The forced flow coupled with heat transfer that is solved in the FEM-model allows for a more closely investigation of the interaction between applied heat transfer and the corresponding gas flow alteration. In Fig 5a, a typical result is depicted of half of the chamber where the arrows show the direction of the flow and the different colors show the temperature. The gas flow is laminar over the substrate. If we look more closely to the flow by zooming in to the hotspot, we can see that just before (Fig. $5 b)$ the laser is switched on, the flow is laminar but right after the laser is switched on (Fig. 5c) a sudden change in streamlines is apparent. This effect is due to the instant temperature increase which results in a localized high pressure affecting the flow. After some time (Fig. 5d), the flow is stabilized again.

\subsection{Nucleation}

The miniaturized reaction chamber has the advantage of being optically accessible. To investigate growth kinetics, a Si-detector is used to collect the reflected laser light. Any growth of carbon nanotubes absorbs laser irradiance, decreasing the reflected laser light. This sensor can be used for investigating certain basic growth characteristics. For instance, the nucleation and onset of the growth is characterized by a sudden drop in the reflection signal. The time it takes for the drop to occur is depending on the temperature. This is shown in Fig. 6. At a certain temperature, the nucleation time does not decrease anymore. This is a consequence of the closed-loop system. The laser has a maximum laser irradiance level and for temperatures above $800^{\circ} \mathrm{C}$ the time it takes to reach the radiation set-point at the maximum laser irradiance exceeds the nucleation time. So the nucleation starts while the laser is still ramping up to the reference temperature. The time to reach the set-point is plotted in red in the figure. Notice this time does not exceed 4 seconds, a consequence of the specific PID-controller characteristics which result in a faster reaching of the set-point, allowing some overshoot to occur. The inset of the figure shows the laser and reflection characteristics for the experiment labeled " 1 " in the figure. The top part of the inset shows a typical laser irradiance response to a certain input reference radiation set-point. The drop of the laser irradiance shows the set-point is (almost) reached ensuring the radiation to remain constant. The resulting reflection is also characterized by a drop, indicating the start of the growth process.

For temperatures below the threshold of $800^{\circ} \mathrm{C}$, it is possible to make an Arrhenius plot where the inverse of the temperature is plotted versus the natural logarithm of the nucleation rate. Arrhenius law states that a process can be characterized by a rate constant $k$ which is given by,

$$
k(T)=v e^{\left(E_{a} / R T\right)}
$$

Here, $v$ is the prefactor which is reaction dependent, $E_{a}$, the activation energy, $R$ the universal gas constant and $T$ the temperature. In this case we define the nucleation rate as 1 over the nucleation time. The slope can be calculated by taking the natural logarithm of the rate and plotting it against the inverse of calculated temperature. The results are presented in Fig 7. The linear fit of the part below $800^{\circ} \mathrm{C}$ is shown as a red line. From the slope of this line we can extract the apparent activation energy for this process. 


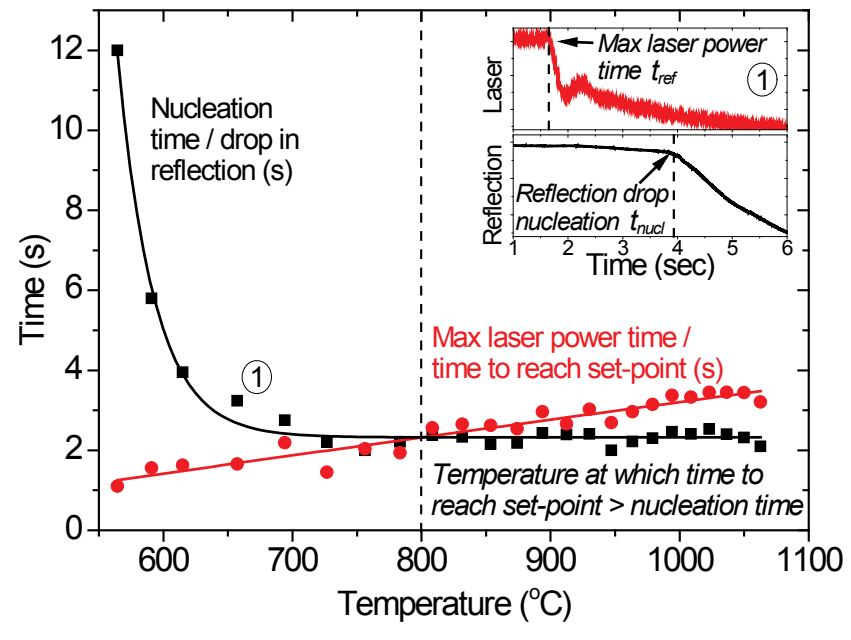

Fig. 6 Graph comparing nucleation time to reference temperature. Also depicted is the time to reach to reference temperature, which is equal to the time the laser irradiates at maximum. When that time exceeds the nucleation time, the nucleation time does not decrease anymore. The inset shows the laser and signal response for experiment 1 . Indicated are the max laser power time and the nucleation time.

The apparent activation energy is calculated to be $0.60 \mathrm{eV}$. This is lower than the reported activation energy for classical thermal CVD where activation energies between 0.8 and $2.0 \mathrm{eV}$ were found which is closer to the activation energy of carbon diffusion in bulk $\gamma$-iron [10-14]. However, it still is higher than the surface diffusion limited growth reported by Hoffman et al. where an activation energy of $0.23 \mathrm{eV}$ was found [15]. We recently reported activation energies depending on the rate-limiting mechanism in the range of $0.3-0.8 \mathrm{eV}$ for laser-assisted chemical vapor deposition [16]. For similar experimental conditions as described here, we found an activation energy of $0.8 \mathrm{eV}$. However, those activation energies are calculated from measuring the growth rate of the nanotubes, instead of measuring the onset of growth, as we did in this study. Since the nucleation is not only depending on the diffusion and dissociation terms of the growth kinetics but also on the rate of critical nuclei formation, it could be argued that a discrepancy can be the result of the different interpretation of processes during nucleation. Another reason could be the overshoot in temperature during heating to the reference radiation set-point, which is not yet fully understood. As a consequence, higher temperatures then assumed here, would result in the estimation of a larger activation energy. Finally, the small amount of data used to fit the Arrhenius equation could account for large errors, in other research as well as here [12].

To validate the experimentally obtained activation energy, the results from the thermal model are used to evaluate the internal energy of the process around the laser hot spot. For the different experiments this internal energy is evaluated at the time the nucleation starts (see Fig 6). The internal energy is calculated by multiplying it by the molar mass of the iron catalyst. The values obtained from the thermal model range from $0.52 \mathrm{eV}$ to $0.62 \mathrm{eV}$.

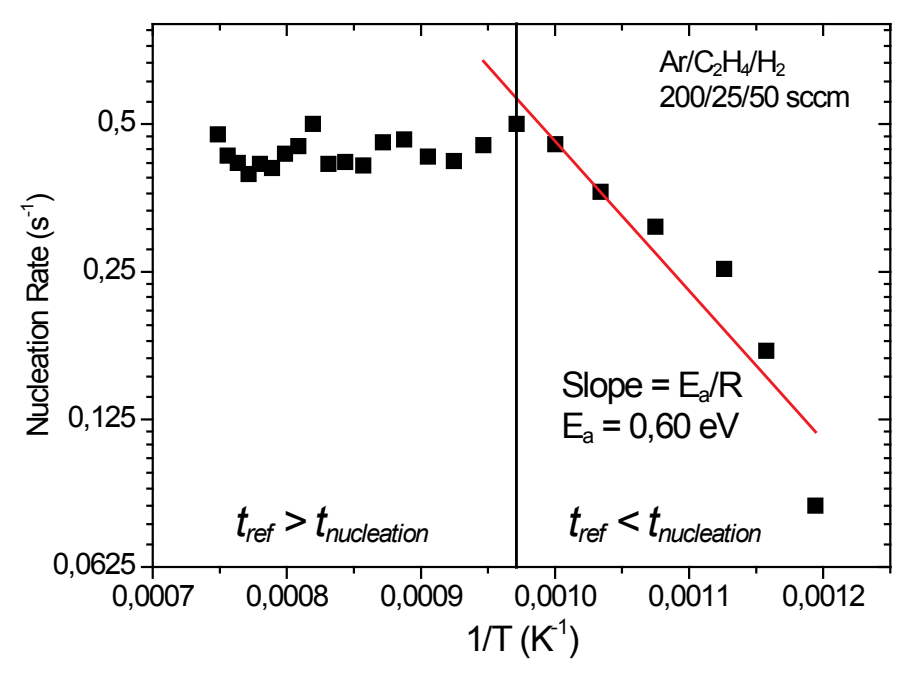

Fig. 7 Arrhenius plot of the nucleation of the process where the inverse of the temperature is plotted against the nucleation rate, the inverse of the nucleation time. Above a certain temperature the time to reach the reference temperature exceeds the nucleation time, so the rate doesn't increase anymore.

\section{Applications}

One of the versatilities of laser-assisted CVD growth of carbon nanotubes is the ability to grow at surfaces, substrates and on devices that already contain components. These components can even be temperature sensitive. Another advantage is the ability to write patterns and lines without having to pre-pattern the catalyst. To demonstrate this we have produced both nanotubes in a narrow enclosure as well as a line of nanotubes by moving the complete chamber with respect to the beam.

\subsection{Growth in a narrow enclosure}

We used the laser to grow carbon nanotubes on the substrate in a narrow enclosure. Taking into account the heat resistance of this enclosure, it is possible to use any material. One can think of electronic components that already surround the position where carbon nanotube growth is desirable. Also polymers such as PDMS can be used as long as the growth is far away enough to not damage the polymer. In this case we have created U-shaped patterned piece of fused silica by femto-second laser machining in combination with an etch process. A schematic of this is part on top of the substrate is shown in Fig 8A. The fused silica part is attached to the silicon
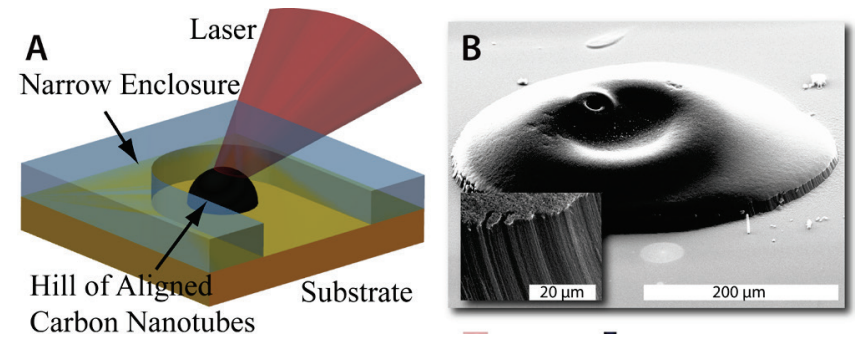

Fig. 8 Demonstration of growth in a narrow enclosure. A. Schematic of the process. B. SEM picture showing the resulting hill of aligned carbon nanotubes. Inset shows a zoom of the side of the hill clearly indicating the aligned nanotubes. 


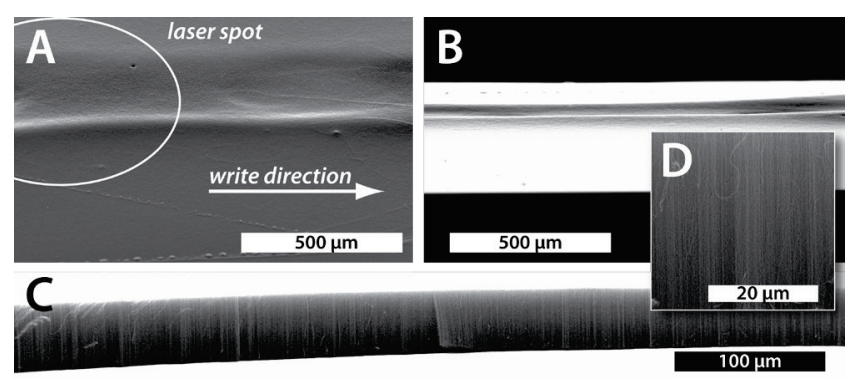

Fig. 9 SEM pictures of a line of aligned nanotubes grown by moving the complete reaction chamber under the laser. A. Overview including an indication of the laser spot size and the write direction. Inset shows the Raman intensity signal and indicates multiwalled nanotubes. B. Side view. C. Side view after scratching the surface. D. Zoom of a part of the aligned nanotubes.

substrate by a heat resistant epoxy. The gas flow is from the right side, into the U-shape. In Fig $8 \mathrm{~B}$ an SEM picture shows the resulting hill of aligned nanotubes that was grown. The inset of the figure shows a zoom of the side of the hill were the aligned nanotubes are clearly visible. Despite the major thermodynamic changes introduced by the addition of such part, the closed-loop system combined with a controlled gas flow results in a controlled growth of aligned nanotubes.

\subsection{Writing lines}

The miniaturized reaction chamber is attached to an $\mathrm{x}$ $y-z$ table allowing us to move the chamber during the synthesis of nanotubes. As a demonstration of this we have grown a line of aligned nanotubes by moving the complete chamber under the laser for about $1.5 \mathrm{~mm}$. The resulting growth is shown in Fig 9. In Fig 9A an overview of the line is shown including an indication of the laser spot size and growth direction. In Fig 9B and $\mathrm{C}$ the line is visualized from the side while the latter is after scratching the surface, clearly showing the aligned nanotubes. In Fig 9D a part of this is zoomed to show the alignment better.

\section{Conclusion}

This paper shows the versatility of the miniaturized reaction chamber. A direct result from the laser-assisted growth process is the fast heating rate and the radiation feedback control allows us to prevent overheating. A precise knowledge of gas composition during synthesis is now available due the forced laminar flow over the substrate. Thanks to a FEM thermal model the temperature is known with an accuracy of within 13\% in open loop. With this information we can investigate nucleation kinetics and corresponding apparent activation energy. An additional result of the small reaction chamber is the much shorter experimentation time and the ability to move the chamber under laser irradiance creating lines and patterns as demonstrated.

\section{References}

[1] W. A. de Heer, A. Chatelain, and D. Ugarte, Science 270, 1179 (1995).

[2] R. Martel, T. Schmidt, H. R. Shea, T. Hertel, and P. Avouris, Applied Physics Letters 73, 2447 (1998).
[3] A. Javey, J. Guo, Q. Wang, M. Lundstrom, and H. Dai, Nature 424, 654 (2003).

[4] A. Srivastava, O. N. Srivastava, S. Talapatra, R. Vajtai, and P. M. Ajayan, Nat Mater 3, 610 (2004).

[5] A. Naeemi and J. D. Meindl, Annual Review of Materials Research 39, 255 (2009).

[6] J. Kong, N. R. Franklin, C. Zhou, M. G. Chapline, S. Peng, K. Cho, and H. Dai, Science 287, 622 (2000).

[7] Y. van de Burgt, Y. Bellouard, R. Mandamparambil, M. Haluska, and A. Dietzel, Journal of Applied Physics 112, 034904 (2012).

[8] M. Haluška, Y. Bellouard, Y. van de Burgt, and A. Dietzel, Nanotechnology 21, 075602 (2010).

[9] D. B. Geohegan, A. A. Puretzky, I. N. Ivanov, S. Jesse, G. Eres, and J. Y. Howe, Applied Physics Letters 83, 1851 (2003).

[10] C. T. Wirth, C. Zhang, G. Zhong, S. Hofmann, and J. Robertson, ACS Nano 3, 3560 (2009).

[11] J. B. In, C. P. Grigoropoulos, A. A. Chernov, and A. Noy, ACS Nano 5, 9602 (2011).

[12] D. S. Engstrøm, N. L. Rupesinghe, K. B. K. Teo, W. I. Milne, and P. Bøgild, Journal of Micromechanics and Microengineering 21, 015004 (2011).

[13] M. J. Bronikowski, J. Phys. Chem. C 111, 17705 (2007).

[14] V. Jourdain and C. Bichara, Carbon 58, 2 (2013).

[15] S. Hofmann, G. Csányi, A. C. Ferrari, M. C. Payne, and J. Robertson, Phys. Rev. Lett. 95, 036101 (2005).

[16] Y. van de Burgt, Y. Bellouard, and R. Mandamparambil, Phys. Chem. Chem. Phys. 16, 5162 (2014).

(Received: July 2, 2013, Accepted: April 10, 2014) 